\title{
Deletion of the glycosyltransferase bgsB of Enterococcus faecalis leads to a complete loss of glycolipids from the cell membrane and to impaired biofilm formation
}

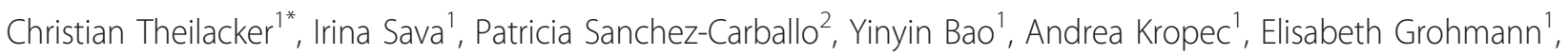
Otto Holst ${ }^{2}$ and Johannes Huebner ${ }^{1}$

\begin{abstract}
Background: Deletion of the glycosyltransferase bgsA in Enterococcus faecalis leads to loss of diglucosyldiacylglycerol from the cell membrane and accumulation of its precursor monoglucosyldiacylglycerol, associated with impaired biofilm formation and reduced virulence in vivo. Here we analyzed the function of a putative glucosyltransferase EF2890 designated biofilm-associated glycolipid synthesis B (bgsB) immediately downstream of bgsA.

Results: A deletion mutant was constructed by targeted mutagenesis in E. faecalis strain 12030. Analysis of cell membrane extracts revealed a complete loss of glycolipids from the cell membrane. Cell walls of $12030 \Delta b \mathrm{gsB}$ contained approximately fourfold more LTA, and ${ }^{1} \mathrm{H}$-nuclear magnetic resonance (NMR) spectroscopy suggested that the higher content of cellular LTA was due to increased length of the glycerol-phosphate polymer of LTA. $12030 \triangle$ bgs $B$ was not altered in growth, cell morphology, or autolysis. However, attachment to Caco-2 cells was reduced to $50 \%$ of wild-type levels, and biofilm formation on polystyrene was highly impaired. Despite normal resistance to cationic antimicrobial peptides, complement and antibody-mediated opsonophagocytic killing in vitro, $12030 \Delta$ bgsB was cleared more rapidly from the bloodstream of mice than wild-type bacteria. Overall, the phenotype resembles the respective deletion mutant in the bgsA gene. Our findings suggest that loss of diglucosyldiacylglycerol or the altered structure of LTA in both mutants account for phenotypic changes observed.

Conclusions: In summary, BgsB is a glucosyltransferase that synthesizes monoglucosyldiacylglycerol. Its inactivation profoundly affects cell membrane composition and has secondary effects on LTA biosynthesis. Both cell-membrane amphiphiles are critical for biofilm formation and virulence of E. faecalis.
\end{abstract}

\section{Background}

The properties of the bacterial cell envelope are pivotal for the interaction of bacteria and the host organism [1]. Enterococcus faecalis expresses several cell-wall glycopolymers that make up the cell envelope, including capsular polysaccharides [2], cell-wall carbohydrates [3], cellwall teichoic acid, lipoteichoic acid (LTA) [4], and glycolipids [5]. We have recently constructed a deletion mutant of the glycosyltransferase bgsA in E. faecalis [5].

\footnotetext{
* Correspondence: christian.theilacker@uniklinik-freiburg.de

${ }^{1}$ Center for Infectious Diseases and Travel Medicine, University Medical

Center Freiburg, Germany

Full list of author information is available at the end of the article
}

Deletion led to a profound shift of the equilibrium of the two main cell wall glycolipids: monoglucosyldiacylglycerol (MGlcDAG) accumulated in the cell membrane of the bgsA mutant, while the production of diglucosyldiacylglycerol (DGlcDAG) was completely abrogated [5]. The bgsA mutant displayed normal cell morphology and growth characteristics but was impaired in attachment to colonic epithelial cells, and biofilm formation was almost completely abolished [5]. Remarkably, the LTA content of the mutant was higher due to the increased length of the glycerol-phosphate polymer.

The role of glycolipids in membrane physiology has been investigated in the cell wall-less bacterium

\section{Ciomed Central}


Acholeplasma laidlawii, which produces glycolipids that are chemically identical to MGlcDAG and DGlcDAG of E. faecalis [6,7]. In Acholeplasma, the ratio of DGlcDAG to MGlcDAG governs the lipid bilayer's elasticity, curvature, and surface-charge density [6-8]. Interestingly, the pathway of glycolipid synthesis is highly conserved, and the type 4 family of NDP-glucose glycosyltransferases contains 107 UDP-sugar glycosyltransferases of bacterial, fungal, and plant origin [9]. Aside from their role as cell membrane components, glycolipids are also involved in the synthesis of LTA in bacteria with low $\mathrm{G}+\mathrm{C}$ content [10]. LTA has a number of important functions in bacterial physiology including cation homeostasis, resistance to antimicrobial peptides, autolysin activity, non-covalent anchoring of cell-surface proteins, attachment to host tissues, and biofilm formation [1,11]. Glycolipids also function as acceptors of the glycerol-phosphate polymer during LTA synthesis, although the exact mechanism underlying this process is still under investigation [10]. If the processive glycosyltransferase YpfP is inactivated in Staphylococcus aureus, DAG instead of DGlcDAG is utilized as a building block in LTA synthesis, suggesting that glycolipids are not essential acceptors of the LTA polymer [12,13].

A second glycosyltransferase (EF 2890) is located immediately downstream of $b g s A$. To our knowledge, the function of this gene locus of E. faecalis or its homologues in streptococci is still unknown. In the current study, we report the construction of a deletion mutant of EF_2890 that we designated $\operatorname{bgs} B$ and studied the role of glycolipid metabolism in LTA biosynthesis and bacterial physiology.

\section{Results}

Construction of a deletion mutant of the glycosyltransferase $b g s B$

Immediately downstream from $\operatorname{bgs} A$, we identified a putative 1,2-diacylglycerol 3-glucosyltransferase (TIGR number EF2890) by basic local alignment search tool (BLASTP) search (Figure 1). This glycosyl-transferase shows homology to YP_001620482.1 of Acholeplasma laidlawii (identity 34\%, similarity 55\%) [14] and to Lmo2555 of Listeria monocytogenes (identity 23\%, similarity 41\%) [15]. We designated this gene bgsB. To study the requirement of $b g s B$ for glycolipid production, LTA synthesis, and bacterial physiology, we constructed a deletion mutant by targeted mutagenesis using the strategy previously applied for the $b g s A$ deletion mutant. Unmarked deletions were created by allelic exchange, and all gene deletions were confirmed by PCR. In the resulting mutant, an internal fragment of 790 bp was deleted from the $b g s B$ gene (Figure 1). Single gene reconstitution of bgsB in E. faecalis $12030 \Delta$ bgsB completely restored the wild-type phenotype, including the glycolipid expression profile in cell membrane extracts (Figure 2) and biofilm formation (Figure 3).

\section{Deletion of $\operatorname{bgs} B$ leads to a complete loss of glycolipids} from the cell membrane and to expression of LTA with increased chain length

We hypothesized that, because it is located immediately downstream from $b g s A$ and has high homology to ALmgs in Acholeplasma laidlawii, the gene product of bgsB glycosylates diacylglycerol to yield MGlcDAG. To test this hypothesis, we extracted the total lipids of the

A

C

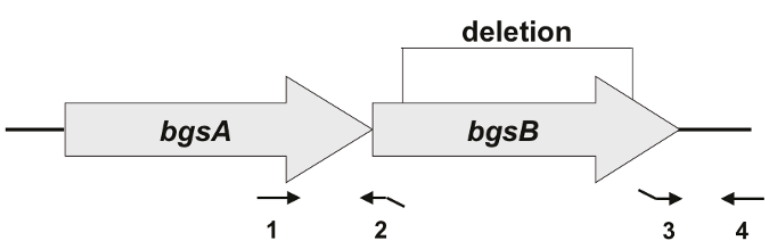

Monoglycosyl-diacylglycerol (MGIcDAG)

B
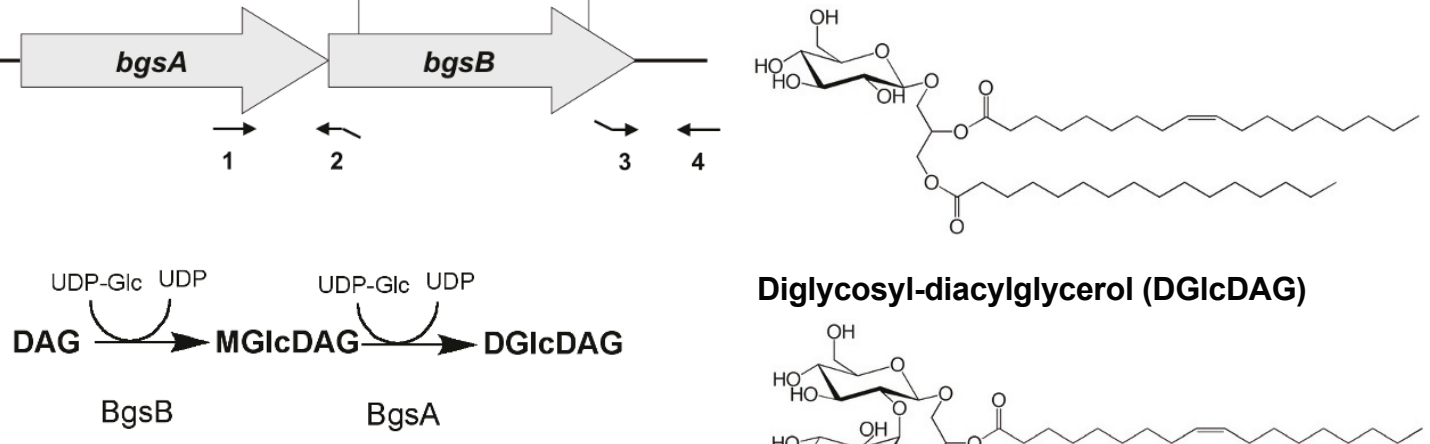

Diglycosyl-diacylglycerol (DGIcDAG)

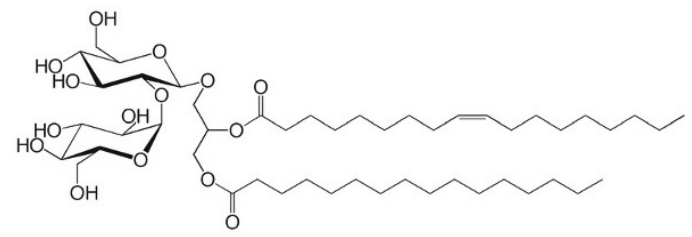

Figure 1 Biosynthesis of glycolipids in E. faecalis. A Genetic organization of the bgs-locus in E. faecalis. The numbers refer to the primers described in Table 2. bgsB has a length of $1224 \mathrm{bp}$. A putative transcriptional terminator is found 10 bases downstream of bgsB. B Putative biosynthetic pathway of glycolipid synthesis in E. faecalis. C Structure of E. faecalis glycolipids. The position of 18:1 and 16:0 fatty acids has not been determined [5]. 

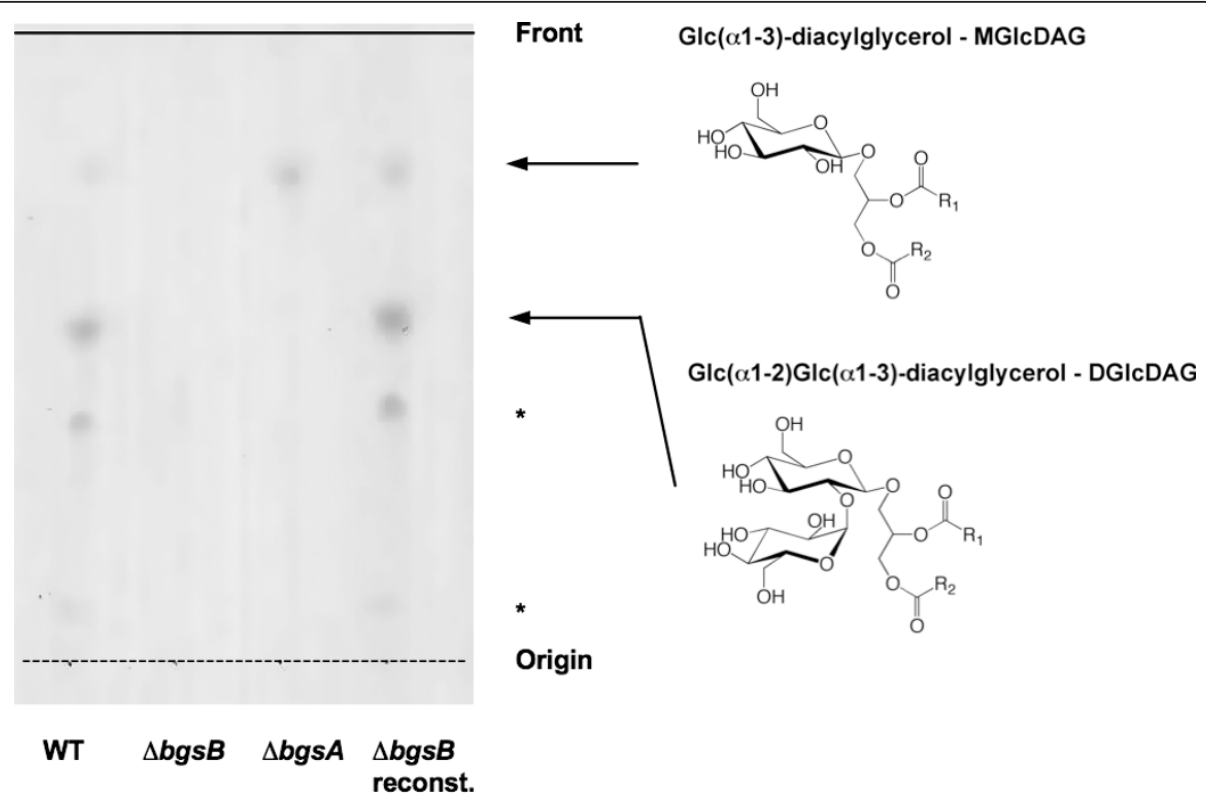

Origin

Figure 2 Thin-layer chromatography of cell-membrane total lipid extracts of $E$. faecalis strains. Bacterial cells were grown overnight, disintegrated, and stirred with butanol. Membrane lipids were extracted from butanol by phase partition according to Bligh and Dyer. $50 \mu \mathrm{g}$ of lipid extract was applied to the TLC plate, separated using a solvent system of $\mathrm{CHCl}_{3} / \mathrm{MeOH} / \mathrm{H}_{2} \mathrm{O}(65: 25: 4, \mathrm{v} / \mathrm{v} / \mathrm{v})$, and visualized with $\alpha$-naphtol/ sulfuric acid. The identity of the bands has been confirmed previously [5]. The glycolipids marked with an asterisk have not been analyzed.

cell membrane, separated them by thin layer chromatography (TLC), and stained glycolipids with $\alpha$-naphthol (Figure 2). As shown previously, inactivation of $b g s A$ resulted in accumulation of MGlcDAG in the cell membrane (Figure 2). In contrast, no glycolipids were visualized in $12030 \Delta b g s B$ extracts, suggesting that $b g s B$ encodes for a glycosyltransferase that glycosylates DAG to form MGlcDAG. MGlcDAG is the substrate of BgsA, which adds a second glucose to yield DGlcDAG (Figure 1). Since BgsA does not accept DAG as a

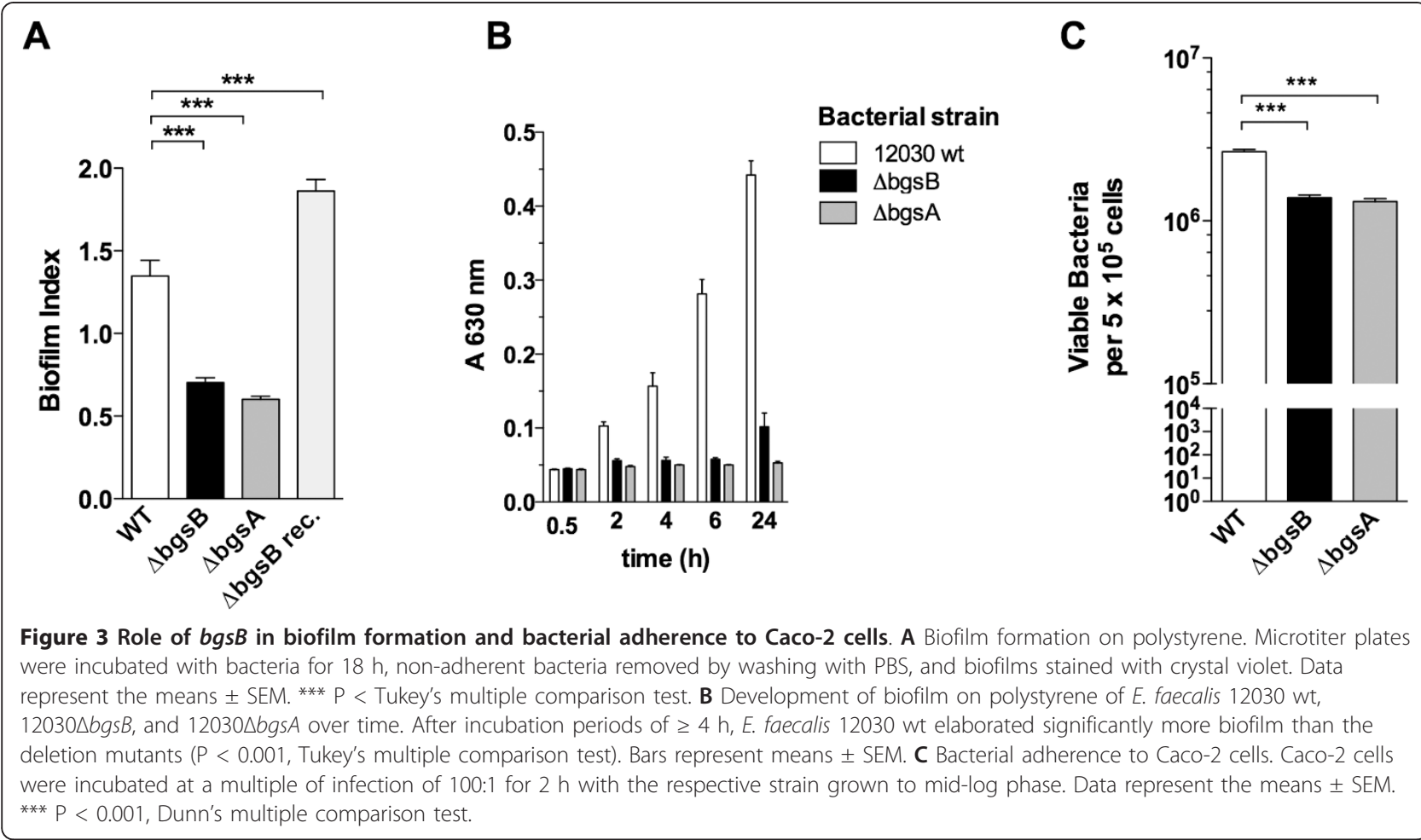


substrate, inactivation of $\mathrm{BgsB}$ results in the loss of all glycolipids from the cell membrane (Figure 2).

We recently showed that inactivation of $b g s A$ also affects LTA synthesis, increasing the chain length of the glycerol-phosphate polymer [5]. Inactivation of $b_{s} B$ has a similar effect on the LTA chain length (Figure 4). To estimate the chain length of the glycerol-phosphate chain by ${ }^{1} \mathrm{H}$-NMR analysis, we used the fatty acid signals of the molecule as an internal reference and compared the integration values of $\mathrm{H} 1$ of glucose and $-\mathrm{CH} 3$ of alanine to the - $\mathrm{CH} 3$ and - $\mathrm{CH} 2$ - signals $(\delta 1.26-1.29$, and 0.88 ) of the fatty acids [5]. The integral ratios yielded higher amounts of glucose and alanine incorporated into the LTA of $12030 \Delta b g s B$ and $12030 \Delta b g s A$ compared to the wild type, suggesting an increased length of the glycerol-phosphate polymer (Figure 4). These results are supported by quantification of LTA from butanol extracts by ELISA (Figure 5). Approximately fourfold more LTA was recovered from butanol extracts of cell walls of $12030 \Delta$ bgs $B$ and $12030 \Delta b g s A$ than from wild-type bacteria. To determine whether increased amounts of LTA were also released into the culture medium, we blotted the culture supernatant onto PVDF membranes and performed semi-quantitative immuno-dot blot analysis (Figure 5). For both mutants, $12030 \Delta b g s B$ and $12030 \Delta b g s A$, increased amounts of LTA in the liquid medium were detected, indicating a higher turnover of LTA in the cell envelope.

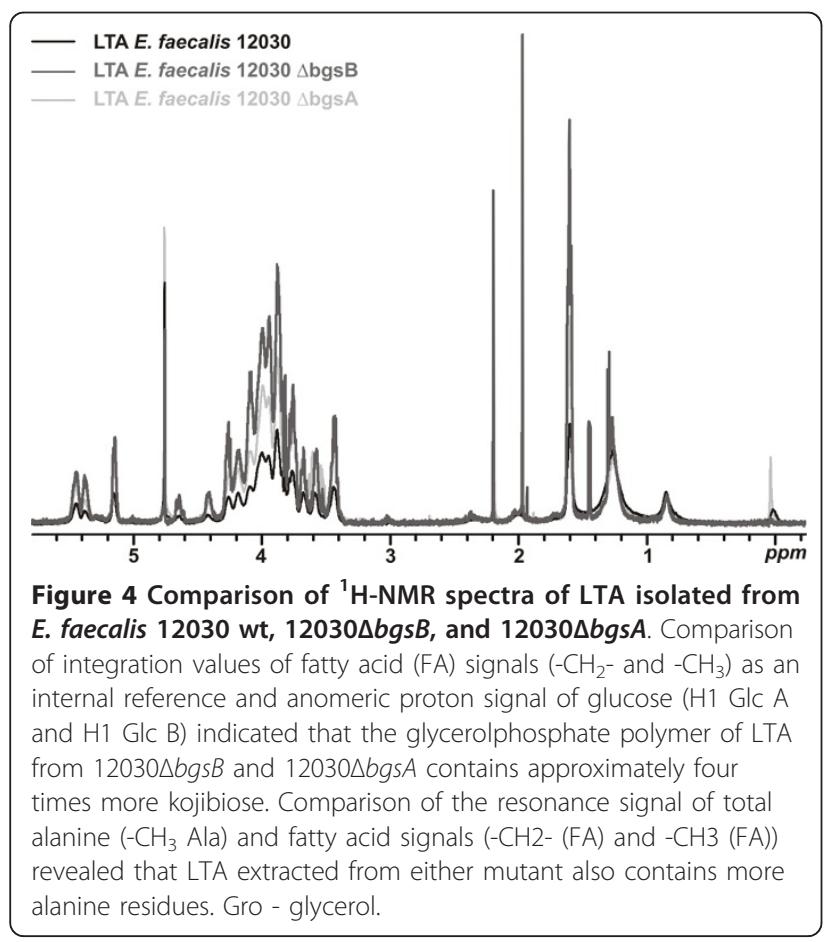

A

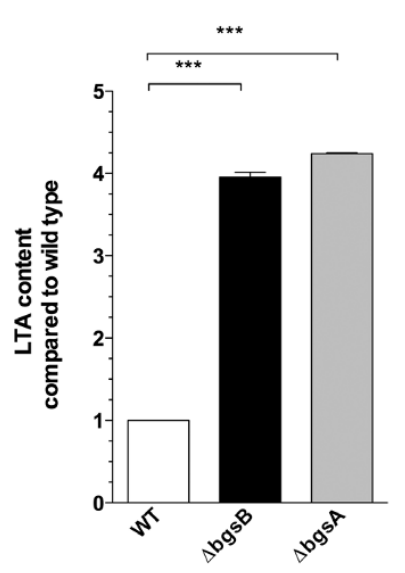

B

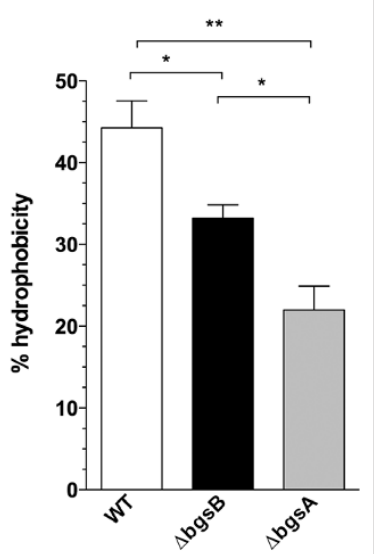

C

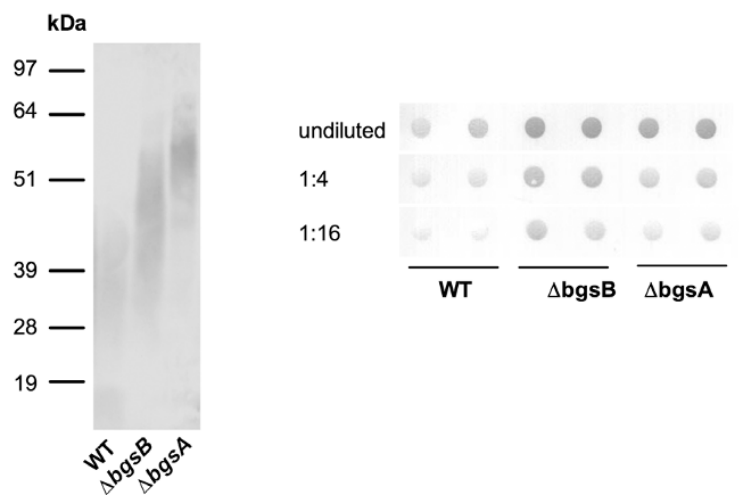

Figure 5 Impact of bgsB on the synthesis and anchoring of LTA in the cell wall and on hydrophobicity of $E$. faecalis cells. A The total amount of butanol-extracted LTA from cell-wall extracts as determined by ELISA. For the quantification of LTA tethered to the cell wall, bacteria were grown overnight and adjusted to the same $\mathrm{OD}_{600}$. Cell walls were disrupted by shaking with glass beads, and LTA was mobilized by stirring bacterial cells with butanol/water ELISA plates were incubated with various concentrations of the respective water phase of the extraction, and LTA was detected using a polyclonal rabbit anti-LTA antibody. Data points represent means \pm SEM, ${ }^{* * *} P<0.001$, Tukey's multiple comparison test. $\mathbf{B}$ Cell-surface hydrophobicity of $E$. faecalis strains determined by adherence of bacterial cells to a mixture of dodecane and aqueous phase. Bars represent the percentage of bacteria remaining in the organic phase after partitioning of the solvent system. Data represent the means \pm SEM, ${ }^{*} P<0.01,{ }^{*} P<0.05$, Tukey's multiple comparison test. C Western blot detection of LTA from 12030 wild type and deletion mutants. LTA was extracted from disrupted bacterial cells after shaking with glass beads by boiling in SDS. LTA was loaded onto $4-12 \%$ SDS PAGE gels and detected using a polyclonal rabbit antibody against enterococcal LTA. D Estimation of LTA shed into the culture medium. After overnight culture, bacterial density was adjusted to the same $\mathrm{OD}_{600}$, and bacteria were removed by centrifugation. $100 \mu \mathrm{l}$ of supernatant was blotted onto PVDF membrane. Bound LTA was detected using the same antibody used in the ELISA. Dilution steps of culture supernatant are indicated in the legend. 
Previous studies in S. aureus and Listeria monocytogenes have shown that substitution of DGlcDAG by MGlcDAG or DAG as the glycolipid anchor of LTA retards the migration of the molecule in SDS-PAGE $[13,15]$. LTA extracted from both mutants displayed a slower mobility in SDS PAGE than wild-type LTA, with LTA from $12030 \Delta b g s B$ migrating faster than LTA from $12030 \Delta$ bgs $A$ (Figure 5). This suggests that both mutants express different lipid anchors from those in the wild type. As DAG is the only substrate available for LTA synthesis in $12030 \Delta b g s B$, it likely serves as lipid anchor in this strain.

LTA and glycolipids are also major determinants of cell-surface charge density. Therefore, hydrophobicity of wild-type and mutant bacteria was determined by measuring the adherence to dodecane. Reduced adherence was observed for both $12030 \Delta$ bgs $A$ and $12030 \Delta b g s B$ (Figure 5). However, $12030 \triangle b g s B$ had higher hydrophobicity than $12030 \Delta b g s A$ ( $44 \%$ wild type versus $33 \%$ $12030 \Delta b g s B$ and $22 \% 12030 \Delta b g s A)$.

\section{Bacterial physiology is not significantly impaired in a bgs $B$ deletion mutant}

Previous studies have shown that LTA and glycolipids play important roles in growth, cell envelope integrity, and cell division [11]. However, despite the complete lack of glycolipids in the cell membrane and increased production of LTA, important characteristics of $12030 \Delta$ bgs B did not differ from wild-type bacteria: Mutants did not differ from wild-type bacteria in their growth kinetics in broth culture (data not shown). Cell morphology of $12030 \Delta$ bgsB determined by transmission electron microscopy was not affected (Additional file 1). Likewise, autolysis was not affected in $12030 \Delta b g s B$ (Additional file 2). Since phosphatidylglycerol from the cell membrane is used as a substrate for polyglycerolphosphate synthesis by LtaS [10], we investigated whether increasing chain length of LTA affects cell membrane content of phosphatidylglycerol in the mutant. However, the semi-quantitative analysis of extracts of total membrane lipids by TLC and staining with molybdenum blue did not reveal differences in phospholipid composition (Additional file 3). The composition and total amount of aminophospholipids as assessed semi-quantitatively by TLC also did not differ between the wild type and $12030 \Delta$ bgsB (Additional file 3 ). Neither did analysis of non-covalently bound surface proteins by SDS-PAGE reveal major differences between the $b_{s s} B$ deletion mutant and the parental strain (Additional file 3).

\section{Deletion of the glucosyltransferase $b g s B$ has no effect on resistance to complement, antimicrobial peptides, and opsonophagocytic killing}

LTA has been shown to be critical for resistance against killing by cationic antimicrobial peptides [1] and has been identified as a target of opsonic antibodies against E. faecalis [4]. To characterize the sensitivity of $12030 \Delta$ bgs $B$ to host defense mechanisms, we assessed its resistance to antimicrobial peptides nisin, polymyxin $B$, and colistin. For nisin, no difference was found between the wild-type and the $\operatorname{bgs} B$ deletion mutant (Additional file 4). A two-fold lower concentration of polymyxin $B$ and colistin was required for killing of $12030 \Delta$ bgs $B$ compared to the isogenic wild type strain. At a serum concentration of $1.7 \%$, sensitivity to complement-mediated phagocytosis did not differ between the 12030 wild type and $12030 \Delta$ bgsB (Additional file 2). Furthermore, rabbit antibodies raised against whole bacterial cells of E. faecalis 12030 mediated opsonophagocytic killing of $12030 \Delta$ bgs $B$ comparable to levels obtained for the wild-type strain (Additional file 2).

\section{The loss of glycolipids from the cell membrane is associated with reduced adherence to Caco-2 cells and impaired biofilm formation}

We recently showed that deletion of $b g s A$ leads to loss of biofilm formation on polystyrene and to reduced adherence to Caco-2 cells [5]. Partial deletion of $b g_{s} B$ also strongly impaired biofilm formation, reducing production by $50 \%$ (Figure 3 ). This defect in biofilm formation was not a result of decreased initial attachment (i. e., bacteria attached in $\leq 30 \mathrm{~min}$ of incubation); rather, it was due to defective accumulation of biofilm mass after initial attachment (Figure 3). Over a period of 24 $\mathrm{h}$, biofilm mass of wild-type bacteria on polystyrene grew in a linear fashion. In contrast, the amount of biofilm produced by $b g s B$ and $b g s A$ mutants remained constant at the level of initial attachment. Adhesion to colonic epithelial cells (Caco- 2 cells) was also impaired in $12030 \Delta$ bgs $B$, reaching only $50 \%$ of the adhesion of wild-type bacteria (Figure 3 )

\section{bgs $B$ contributes to virulence during bacteremia in mice}

Previous experiments with a $b g s A$ deletion mutant in $E$. faecalis showed that it leads to an attenuation of virulence in a mouse bacteremia model [5]. To assess whether cell membrane glycolipids or glycolipid anchoring of LTA is required for the pathogenesis of enterococcal infections, we employed the same model to investigate the bgsB mutant. As mentioned above, 12030 wild-type and respective mutants had comparable growth characteristics. For virulence studies, we infected BALB/c mice 6 - 8 weeks old by i.v. injection, sacrificed the animals after 3 days, and enumerated the viable bacteria. Pilot experiments indicated that, with a high inoculum of $2 \times 10^{9}$ bacteria, infected mice are bacteremic up to 4 days without succumbing to the infection. Compared to the wild type, mice infected with $12030 \Delta$ bgs $B$ or $12030 \Delta$ bgs $A$ cleared significantly more 
bacteria from the bloodstream (Figure 6). No difference in virulence between $12030 \Delta b g s B$ and $12030 \Delta b g s A$ was detected in this model.

\section{Discussion}

Although MGlcDAG and DGlcDAG are the major glycolipids in many Gram-positive bacteria, distinct differences are found between species in biosynthetic pathways. In staphylococci and Bacillus, a single processive glucosyltransferase YpfP adds two glucose residues to DAG to synthesize DGlcDAG $[12,16,17]$. Depending on the bacterial species and strain background, the deletion of this enzyme may result in an increased LTA content and turnover [16], or loss of LTA from the cell membrane, associated with a reduced rate of autolysis and impaired biofilm formation [12]. In listeria, streptococci, and enterococci, genome analysis revealed two putative glycosyltransferases involved in the biosynthetic pathway of glycolipids $[7,14,15,18]$. Homologues of a $(1 \rightarrow 2)$ glucosyltransferase have been investigated in listeria (LafA), group B streptococci (IagA), and E. faecalis (BgsA) $[5,15,18]$. In group B streptococci, deletion of

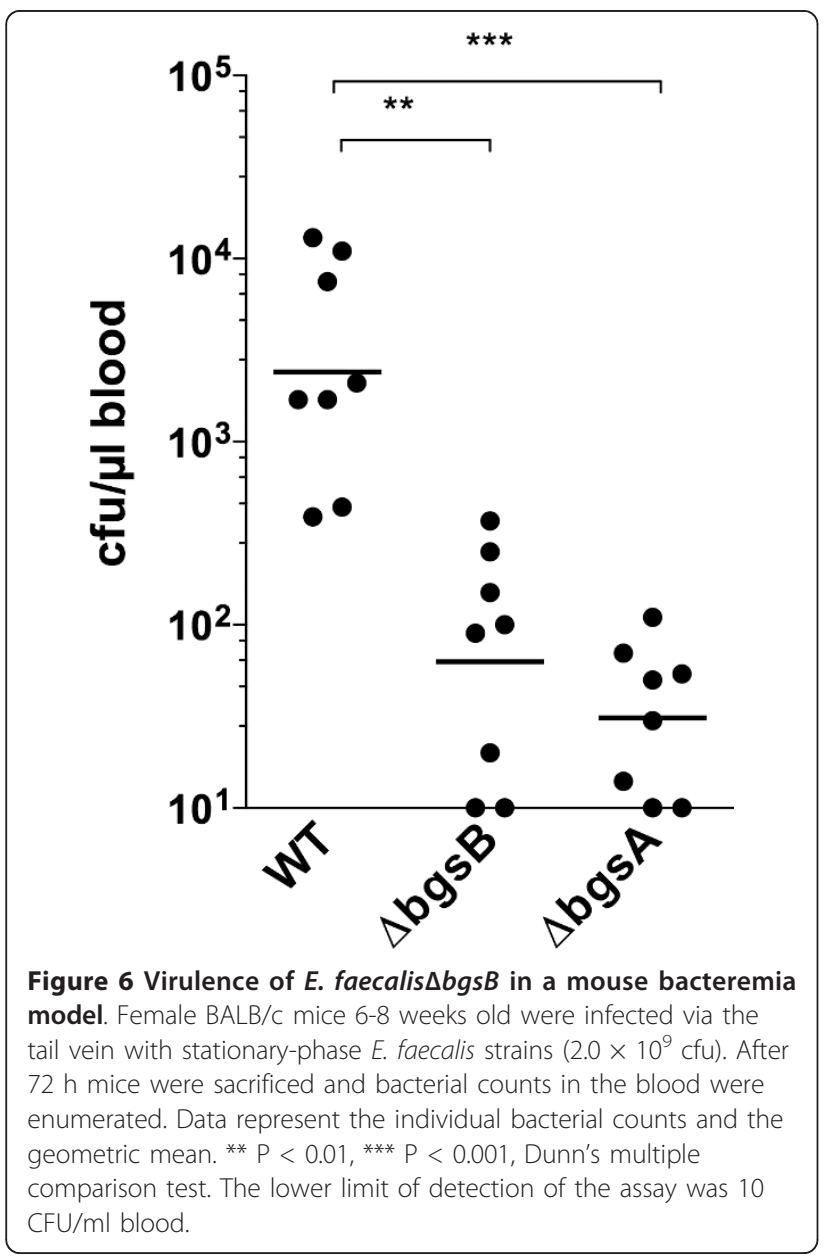

iagA results in the absence of capsule expression, reduced retention of LTA on the bacterial cell surface, and increased release of LTA into the culture medium [18]. Inactivation of lafA in L. monocytogenes strongly depletes LTA from both the cell wall and the culture medium [18]. In contrast to these findings, deletion of bgsA in E. faecalis results in an increased concentration of LTA in the bacterial cell envelope, most likely related to the longer glycerol-phosphate polymer. The different makeup of glycolipids and LTA in this mutant strongly impaired biofilm-formation and affected virulence in vivo [5].

In the current study, we constructed a deletion mutant by targeted mutagenesis of the putative glycosyltransferase $b g s B$ located immediately downstream of bgsA. After inactivation of $b g s B$ in E. faecalis 12030, no glycolipids or glycolipid-derivatives were recovered from the cell envelope of the $12030 \Delta b g s B$ mutant, indicating that $\mathrm{BgsB}$ is a 1,2-diacylglycerol 3-glucosyltransferase. BgsA cannot take the place of BgsB, which suggests that BgsA has higher substrate specificity than YpfP in $S$. aureus and $B$. subtilis $[13,17]$. The putative function assigned to BgsA and BgsB by this work is in agreement with data obtained for their homologues LafA and LafB in L. monocytogenes [15]. Although the lipid anchor of LTA from $12030 \Delta b g s B$ was not characterized chemically, indirect evidence suggests that DAG instead of DGlcDAG anchors LTA to the cell membrane in this mutant. LTA extracted from $12030 \Delta$ bgs $B$ migrated more slowly than wild-type LTA in SDS PAGE, a feature that has been described for homologous LTA molecules substituted with DAG instead of DGlcDAG in $S$. aureus and L. monocytogenes $[13,15]$. In staphylococci and listeria it has been also demonstrated that, in the absence of glycolipids, the enzyme that transfers glycerolphosphate residues to the glycolipid anchor (LtaS) can utilize DAG as glycerolphosphate acceptor for the synthesis of the LTA backbone [13,15]. Deletion mutants of the glucosyltransferases $b g s B$ and $b g s A$ enabled us to study the individual roles of the two major glycolipids MGlcDAG and DGlcDAG in the physiology and virulence of $E$. faecalis. To our surprise, the complete loss of glycolipids from the cell membrane in $12030 \Delta b g s B$ had only minor effects on bacterial morphology, cell growth, and autolysis.

In contrast, MGlcDAG and DGlcDAG are critical for cell membrane elasticity and fluidity and important for the function of membrane-bound proteins in Acholeplasma laidlawii $[6,7,14]$. It is possible, however, that up-regulation of other cell membrane amphiphiles may compensate for the lack of glycolipids in the $\operatorname{bgs} B$ mutant [6]. In fact, the concentration of LTA was increased in $12030 \Delta b g s B$ and possibly compensates for the loss of phosphoglycolipid derivatives of MGlcDAG 
and DGlcDAG in the $12030 \Delta b g s B$ mutant [19]. A characteristic feature of both mutants is the increased chain length of the glycerol-phosphate polymer. However, the mechanism underlying this alteration in LTA structure remains unclear and deserves further attention.

The most notable feature of $12030 \Delta b g s B$ is its impairment in biofilm formation and adherence to colonic cells. As observed previously in the bgsA mutant, initial attachment to polystyrene was not impaired in $12030 \Delta b g s B$, but the accumulation of bacteria in the growing biofilm was impaired. This is in contrast to other biofilm-defective mutants in E. faecalis, in which attachment to the foreign surface is the feature primarily affected and underlines the importance of cell envelope amphiphiles in the retention of bacteria within the biofilm architecture $[20,21]$. Several mechanisms may explain the biofilm phenotype of the mutants. As in the bgs $A$ mutant, impaired biofilm formation in $12030 \Delta b g s B$ was associated with reduced hydrophobicity, a wellknown determinant of biofilm formation in bacteria $[22,23]$. Also, increased LTA concentration in the cell envelope of the $b g s B$-mutant may impair biofilm formation by increasing the net negative charge of the cell envelope. The impact of the higher negative charge of the LTA molecule on biofilm formation has been demonstrated by mutants in the $\mathrm{D}$-alanine-D-alanyl-carrier protein ligase DltA [24,25]. Finally, the increased amount of LTA released into the biofilm matrix (as observed with $12030 \Delta b g s B$ and $12030 \Delta b g s A$ ) may act as a biosurfactant, promoting detachment of bacterial cells from the biofilm and thereby impeding its growth [26]. In contrast to our results the inactivation of the glycosyltransferase YpfP in S. aureus leads to depletion of
LTA from the cell surface and to a reduced ability to form biofilm [12].

Aside from its effects on biofilm formation, the increased density of negative charges of the LTA molecule of the mutant may also explain the slight increase in sensitivity of $12030 \Delta b g s B$ to the antimicrobial peptides colistin and polymyxin $B$. If this difference explains the significantly impaired virulence in our mouse bacteremia model, however, is unclear. On balance, we observed a 2-log reduction in the number of CFU recovered for both mutants, suggesting that glycolipids, either as a cell membrane component or as an anchor of LTA, play a critical role in the cell envelope of enterococci during infection. In general, mutation of the glycosyltransferase $b g s A$ and $b g s B$ yielded similar phenotypes, suggesting that the phenotypic changes observed for both mutants are mainly the result of the depletion of DGlcDAG or altered LTA structure. On the other hand, MGlcDAG seems to play a minor role in bacterial physiology and virulence.

\section{Conclusions}

We have shown that the $b_{s} B$ gene is responsible for the glycosylation of DAG to form MGlcDAG, the first step in glycolipid synthesis in E. faecalis. bgsB deletion led to reduced biofilm formation and attachment to colonic cells, and to impaired virulence in vivo.

\section{Methods}

\section{Bacterial strains, plasmids, and growth conditions}

The bacterial strains and plasmids used in this study are shown in Table 1 . Enterococci were grown at $37^{\circ} \mathrm{C}$ without agitation in tryptic soy broth (TSB; Merck), M17

Table 1 E. faecalis strains and plasmids used in this study

\begin{tabular}{|c|c|c|}
\hline strain or plasmid & characterization & reference \\
\hline \multicolumn{3}{|l|}{ strains } \\
\hline E. faecalis 12030 & Clinical isolate, strong biofilm producer & [33] \\
\hline E. faecalis ATCC 29212 & Reference strain & \\
\hline E. faecalis $12030 \Delta b g s A$ & (EF2891) bsgA mutant & [5] \\
\hline E. faecalis $12030 \Delta$ bgs $B$ & bgs $B$ deletion mutant & This study \\
\hline E. faecalis 12030 $\Delta$ bgsB_rec. & Reconstituted mutant & This study \\
\hline Escherichia coli DH5 $\alpha$ & Gram-negative cloning host & \\
\hline Escherichia coli TOP10 & Gram-negative cloning host & Invitrogen \\
\hline \multicolumn{3}{|l|}{ plasmids } \\
\hline PCASPER & Gram-positive, temperature-sensitive mutagenesis vector & [34] \\
\hline pCRII-TOPO & Gram-negative cloning vector & Invitrogen \\
\hline 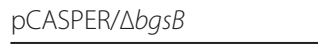 & & This study \\
\hline $\mathrm{pMAD} / \mathrm{bsgB}$ & & This study \\
\hline PMAD & oripE194 ${ }^{\text {ts }}, \mathrm{Em}^{\mathrm{R}}, \mathrm{Amp}^{\mathrm{R}}, \mathrm{bgaB}$ & {$[35]$} \\
\hline
\end{tabular}


broth (Difco Laboratories), or TSB plus 1\% glucose (TSBG) as indicated. In addition, tryptic soy agar or M17 agar plates were used. Escherichia coli DH5 $\alpha$ and TOP10 (Invitrogen) were cultivated aerobically in LBbroth. Kanamycin was added for enterococci $(1 \mathrm{mg} / \mathrm{ml})$ and for E. coli $(50 \mu \mathrm{g} / \mathrm{ml})$; tetracycline was used at 12.5 $\mu \mathrm{g} / \mathrm{ml}$ for $E$. coli and at $10 \mu \mathrm{g} / \mathrm{ml}$ for enterococci.

\section{Construction of a nonpolar deletion mutant of $b g s B$}

Molecular techniques used in this study have been described previously [5]. In brief, the bgsB mutant was constructed in E. faecalis 12030 by homologous recombination. The deletion of a portion of the gene $\operatorname{bgs} B$ (790 bp) (EF_2890 in the E. faecalis V583 genome, GenBank accession no. AAO82579.1) was created as described elsewhere [5]. Primers 1 and 2 (Table 2) were used to amplify a 581-bp fragment downstream, and primers 3 and 4 were used to amplify a 563-bp fragment upstream of the target gene. Primers 2 and 3 contain a 21-bp complementary sequence (underlined in Table 2). Overlap extension PCR was performed to generate a PCR product lacking a fragment of $790 \mathrm{bp}$ in the center of $b g s B$ (Figure 1). The resulting construct was cloned into the Gram-positive shuttle vector pCASPER containing a temperature-sensitive replicon; the resulting plasmid, pCASPER- $\triangle b g s B$, was transformed into E. faecalis 12030 by electroporation. Integrants were selected at the non-permissive temperature $\left(42^{\circ} \mathrm{C}\right)$ on TSA plates with kanamycin. Colonies were passaged between 6 and 10 times in liquid cultures without antibiotics at the permissive temperature $\left(30^{\circ} \mathrm{C}\right)$ and subsequently screened by replica-plating for loss of kanamycin resistance. Kanamycin-sensitive clones were analyzed by PCR for the deleted sequence, and the deletion mutant was designated E. faecalis $12030 \Delta$ bgs $B$.

Complementation of the $12030 \Delta$ bgs $B$ mutant has been done by a knocking in strategy as described previously [27]. Briefly, the $b g s B$ gene $(1224 \mathrm{bp})$ plus $212 \mathrm{bp}$ upstream and 502 bp downstream was amplified using primers 1 and 4, cloned into pCRII-TOPO (Table 1) and digested with EcoRI. The resulting fragment was inserted into plasmid pMAD (Table 1). E. faecalis $12030 \Delta \operatorname{bgs} B$ was transformed with the recombinant

Table 2 Primers used in this study

\begin{tabular}{lll}
\hline & Name & Sequence $\left(\mathbf{5}^{\prime}\right.$-3') \\
\hline 1 & EF2890 & CAAACTGCTCCTTCAGCAACT \\
delF & \\
\hline 2 & EF2890 & ACTAGCGCGGCCGCTTGCTCCCTATTITGTCAGCGCCTCAAC \\
& OEL & \\
\hline 3 & EF2890 & GGAGCAAGCGGCCGCGCTAGTTAGAAGTCGCTACCCCACTCA \\
& OER & \\
\hline 4 & EF2890 & GCGCGACAGTTACCAGAGTAT \\
& delR & \\
\hline
\end{tabular}

plasmid (pMAD-bgsB) and incubated at $37^{\circ} \mathrm{C}$ for $4 \mathrm{~d}$ on TSB plates supplemented with Xgal $(40 \mu \mathrm{g} / \mathrm{ml})$ and erythromycin (Erm, $100 \mu \mathrm{g} / \mathrm{ml}$ ). Dark blue colonies were picked and incubated overnight on fresh plates supplemented with Xgal and Erm at the non-permissive temperature $\left(44^{\circ} \mathrm{C}\right)$. Presence of the wild-type and mutated alleles was determined by PCR, and for each construct the positive clones were cultured in TSB medium supplemented with Erm $(150 \mu \mathrm{g} / \mathrm{ml})$ at $44^{\circ} \mathrm{C}$ over-night. This last step was repeated once, using the overnight culture to inoculate a fresh culture tube. To delete the erythromycin resistance gene, overnight cultures were inoculated in TSB medium without Erm and incubated for $12 \mathrm{~h}$ at $30^{\circ} \mathrm{C}$, followed by $18 \mathrm{~h}$ at $44^{\circ} \mathrm{C}$ without shaking. This step was repeated until white colonies were obtained on Xgal-supplemented TSA plates incubated overnight at $37^{\circ} \mathrm{C}$. Erm sensitivity of the white colonies was verified, and sensitive clones were tested by PCR for the presence of the intact $b_{s s} B$ gene.

\section{Biofilm plate assay}

Enterococci were tested for production of biofilm using a polystyrene microtiter assay [5,24]. Briefly, bacteria were grown at $37^{\circ} \mathrm{C}$ in TSB for $18 \mathrm{~h}$. Polystyrene tissueculture plates (Brandt, Germany) were filled with $180 \mu \mathrm{l}$ of TSB plus $1 \%$ glucose and $20 \mu \mathrm{l}$ of this culture, and the plates were then incubated at $37^{\circ} \mathrm{C}$ for $18 \mathrm{~h}$. The plates were read in an ELISA reader (Bio- $\mathrm{Rad}$ Microplate reader) at an optical density of $630 \mathrm{~nm}$ to assess homogenous growth. The culture medium was discarded, and the wells were washed 3 times with $200 \mu \mathrm{l}$ of PBS without disturbing the biofilm on the bottom of the wells. The plates were dried at $60^{\circ} \mathrm{C}$ for $1 \mathrm{~h}$ and stained with $2 \%$ Hucker's crystal violet for 2 min. Excess stain was removed by rinsing the plates under tap water, and the plates were dried at $60^{\circ} \mathrm{C}$ for $10 \mathrm{~min}$. The optical density at $630 \mathrm{~nm}$ was determined. Biofilm formation was normalized to growth with the biofilm index, which was calculated as OD of the biofilm $\times(0.5 / \mathrm{OD}$ of growth) [24].

\section{Adherence to Caco-2 cells}

Adherence to Caco-2 cells was investigated using methods described previously [28]. In brief, cells were cultivated in DMEM medium supplemented with $10 \%$ fetal bovine serum and 1\% non-essential amino acids under a $5 \% \mathrm{CO}_{2}$ atmosphere. All the experiments were performed on cells between the $15^{\text {th }}$ and $25^{\text {th }}$ passage. Caco-2 cells were cultivated in 24-well plates to a density of $1 \times 10^{5}$ cells/well for 3-5 days. Bacteria were grown to mid-log phase at $37^{\circ} \mathrm{C}$ without agitation in tryptic soy broth; Caco- 2 cells were incubated with bacteria for $2 \mathrm{~h}$ at a multiplicity of infection of 100:1. After infection of the monolayer, epithelial cells were washed 
and lysed with $0.25 \%$ Triton- $\mathrm{X}$ at $37^{\circ} \mathrm{C}$ for $20 \mathrm{~min}$ and adherent bacteria enumerated by quantitative bacterial counts. Pilot experiments had shown no significant bacterial invasion under the outlined conditions.

\section{Isolation and analysis of glycolipids and LTA}

Bacterial cells were resuspended in $0.1 \mathrm{M}$ citrate buffer $\mathrm{pH} 4.7$ and cell walls disrupted by shaking with an equal volume of glass beads $(0.1 \mathrm{~mm}$ glass beads, $3 \times$ 1 min intervals using a BeadBeater, Glenn Mills, Clifton, NJ). Glass beads were removed by sedimentation, and disrupted cells were stirred with an equal volume of $n$ butanol for $30 \mathrm{~min}$. After phase separation by centrifugation, the aqueous layer was removed, dialyzed against $0.1 \mathrm{M}$ ammonium acetate ( $\mathrm{pH} 4.7)$ and lyophilized. LTA was purified from the aqueous phase by hydrophobic interaction chromatography [4]. The butanol phase was evaporated under a vacuum, and cell membrane lipids were extracted according to the method of Bligh and Dyer and separated by TLC $\left(0.2 \mathrm{~mm}\right.$ Silica gel $60 \mathrm{~F}_{254}$ Merck, Darmstadt) using a solvent system of $\mathrm{CHCl}_{3}$ / $\mathrm{MeOH} / \mathrm{H}_{2} \mathrm{O}(65: 25: 4, \mathrm{v} / \mathrm{v} / \mathrm{v})$ and detection with $\alpha-$ naphthol (3.2\%). For detection of phospholipids, TLC plates were stained with molybdenum blue; amino phospholipids were stained with ninhydrin, as previously described [29]. LTA was also analyzed by SDS-PAGE as described previously [5]. Briefly, bacterial cell walls were disrupted by shaking with glass beads as described above, boiled in sample buffer containing SDS, and subjected to SDS-PAGE in gradient gels containing acrylamide $(4 / 12 \% \mathrm{w} / \mathrm{v}$, Invitrogen). Separated LTA was transferred onto PVDF membrane and blocked at $4^{\circ} \mathrm{C}$ in Tris-buffered saline (TBS) containing skim milk (5\% w/ v) for $18 \mathrm{~h}$, then incubated at $20-22^{\circ} \mathrm{C}$ for $2 \mathrm{~h}$ with rabbit antibody raised against $E$. faecalis LTA (see below) diluted 1:200 in TBS/skim milk. After washing in TTBS (Tween $200.05 \% \mathrm{v} / \mathrm{v}$ in TBS), the sheets were incubated at $20-22^{\circ} \mathrm{C}$ for $1 \mathrm{~h}$ with a goat anti-rabbit IgG (whole cell) alkaline phosphatase conjugate (Sigma), diluted 1:1000 with TBS/skim milk, and then washed again in TTBS. Binding of the enzyme-conjugated antibodies was detected with the NBI/BCIP (Biorad). For visualization of proteins, SDS PAGE gels were stained with Coomassie blue.

\section{Characterization of LTA}

Lipoteichoic acid of E. faecalis strain $12030 \Delta b g s B$ was analyzed by NMR spectroscopy as described previously [5].

\section{Rabbit antiserum against LTA}

A female New Zealand White rabbit was immunized s.c. with $100 \mathrm{mg}$ of LTA purified from E. faecalis strain 12030 suspended in complete Freund adjuvant (Sigma), followed by the same dose s.c. suspended in incomplete
Freund adjuvant (Sigma) on day 7. The rabbit was boosted intravenously with three 10-mg doses over the following 3 weeks. After the last vaccination, the rabbit was sacrificed and exsanguinated to obtain the serum.

\section{Autolysis assay and sensitivity to antimicrobial peptides} Cell autolysis was determined as described by Qin et al. [30]. The MIC of polymyxin B, nisin, and colistin against wild-type and $12030 \Delta$ bgs $B$ were determined by a modified NCCLS broth dilution method [24].

\section{Determination of hydrophobicity}

Hydrophobicity was determined by measuring adherence to dodecane [31]. Briefly, bacteria were grown to logarithmic phase and resuspended in sodium phosphate to yield an $\mathrm{OD}_{600}$ of 0.4-0.5. The same volume of dodecane was added, and phases were vigorously vortexed for $1 \mathrm{~min}$, then for $10 \mathrm{~min}$ to allow phase separation. Absorbance of the water-phase was measured. The proportion of cells in the dodecane phase was calculated according to the formula: $\%$ hydrophobicity $=[1-(\mathrm{A} / \mathrm{A} 0)] \times 100$.

\section{Mouse bacteremia model}

The virulence of E. faecalis strain $12030 \Delta$ bgsB was evaluated in a mouse bacteremia model $[5,32]$. In summary, eight female BALB/c mice 6-8 weeks old were challenged by i.v. injection of E. faecalis strains grown to stationary phase $\left(2.0 \times 10^{9} \mathrm{cfu}\right)$ via the tail vein. Seventy-two hours after infection, the mice were sacrificed and exsanguinated, and bacterial counts in the blood were enumerated by serial dilutions. All animal experiments were performed in compliance with the German animal protection law (TierSchG). The mice were housed and handled in accordance with good animal practice as defined by FELASA and the national animal welfare body GV-SOLAS. The animal welfare committees of the University of Freiburg (Regierungspräsidium Freiburg Az 35/9185.81/G-07/15) approved all animal experiments.

\section{Transmission electron microscopy (TEM)}

Bacterial cells were prepared for TEM as described previously [24].

\section{Opsonophagocytic killing assay}

An opsonophagocytic killing assay was used as previously described [5]. In summary, white blood cells (WBC) were prepared from fresh human blood collected from healthy adult volunteers. Using trypan blue staining to differentiate dead from live leukocytes, the final cell count was adjusted to $2.5 \times 10^{7} \mathrm{WBC}$ per ml. Baby rabbit serum (Cedarlane Laboratories, Hornby, Ontario, Canada), diluted 1:15 in RPMI plus $15 \%$ fetal bovine serum (FBS) and absorbed with the target strain, was 
used as complement source. Bacteria cultured on agar plates were resuspended in TSB to an $\mathrm{OD}_{600}$ of 0.1 and then grown to an OD of 0.4. A final 1:100 dilution was made in RPMI-FBS. Equal amounts of PMN and bacteria (multiple of infection 1:1), complement, and heat-inactivated rabbit immune serum were incubated at $37^{\circ} \mathrm{C}$ for $90 \mathrm{~min}$. For the controls, antibody, complement, or PMN were replaced by RPMI-FBS. For enumeration of surviving bacteria, the content of tubes was diluted in TSB, and samples were plated onto tryptic soy agar plates. The percentage of opsonophagocytic killing was calculated by determining the ratio of the CFU surviving in the tubes with bacteria, leukocytes, complement, and antibody to the CFU surviving in the tubes with all these components but lacking leukocytes.

\section{Quantification of LTA}

The LTA content of bacterial cell walls was measured according to the method of Fedtke et al. [12]. In summary, wild-type and mutant bacteria were grown for $18 \mathrm{~h}$ in TSB, adjusted to an equal $\mathrm{OD}_{600}$, and bacteria from equal volumes were collected by centrifugation. Bacterial were disrupted by shaking with glass beads as described above, and LTA was extracted from the cell walls by stirring them in an equal volume of butanol and $0.1 \mathrm{M} \mathrm{Na}$-acetate buffer ( $\mathrm{pH} 4,7$ ). The aqueous phase of the extract was dialyzed, lyophilized, and resuspended in the same volume of phosphate buffer $(\mathrm{pH}$ 7.0). ELISA plates (Brandt) were coated with a range of LTA dilutions at $4^{\circ} \mathrm{C}$ for $18 \mathrm{~h}$, and adherent LTA was detected using a rabbit antiserum specific for E. faecalis LTA as primary antibody (see above). A goat anti-rabbit IgG whole-molecule alkaline phosphatase conjugate (Sigma) served as secondary antibody [5]. LTA from E. faecalis 12030 , purified by hydrophobic-interaction chromatography, was used as a standard. The amount of LTA shed into the culture medium was measured semiquantitatively by immuno-dot-blot analysis. To this end, bacteria were grown in TSB at $37^{\circ} \mathrm{C}$ for $18 \mathrm{~h}$ and adjusted to the same $\mathrm{OD}_{600}$. Bacterial cells were removed by centrifugation, culture supernatant was passed through a $0.45 \mu \mathrm{m}$ membrane filter, and $100 \mu \mathrm{l}$ of supernatant was spotted in various dilutions onto PVDF membrane using a dot-blot microfiltration apparatus (Bio-Dot, Biorad Laboratories, Munich, Germany). The membranes were allowed to dry overnight. Staining of immuno-dot-blots was performed using the same protocol as described for western blot analysis of LTA.

\section{Statistical Methods}

Comparisons were made by one-way ANOVA and Tukey's multiple comparison test (parametric data) or Kruskal-Wallis test and Dunn's multiple comparison test (nonparametric data) as indicated using the Prism Graphpad 4 software package. A p-value of $<0.05$ was considered statistically significant.

\section{Additional material}

\begin{abstract}
Additional file 1: Transmission electron microscopy of $E$. faecalis strains. E. faecalis 12030 wild type (A) and 12030 $\triangle$ bgsB (B). Bar represents $500 \mathrm{~nm}$.

Additional file 2: Autolysis and opsonization of $E$. faecalis $12030 \Delta$ bgs $B$. A Spontaneous bacterial autolysis. Cells were grown to mid-log phase, resuspended in $10 \mathrm{mM}$ sodium phosphate buffer containing $5 \%$ Triton X-100 and the decrease of the OD 600 at $30^{\circ} \mathrm{C}$ was recorded over time. $\mathbf{B}$ Bacterial killing in vitro after $90 \mathrm{~min}$ in the presence of $6.5 \%$ rabbit complement (white bar), $2 \times 10^{7}$ human PMN plus complement (gray bar) and rabbit antiserum raised against whole bacterial cells (serum dilution 1:2500) plus PMN and complement (black bar). Bars represent means \pm SEM.

Additional file 3: Characterization of $E$. faecalis $\Delta b g s B$ cell walls. A Thin-layer chromatography of cell membrane total lipid extracts of $E$. faecalis 12030 wild type (lane 1 and 4), 12030 $\Delta$ bgsB (lane 2 and 5), 12030 $\triangle$ bgsA (lane 3 and 6). TLC plates were developed using a solvent system of $\mathrm{CHCl}_{3} / \mathrm{MeOH} / \mathrm{H}_{2} \mathrm{O}(65: 25: 4, \mathrm{v} / \mathrm{v} / \mathrm{v})$. Staining lane 1 - 3 molybdenum blue, lane 4 - 6 ninhydrin. B SDS PAGE of bacterial whole protein extracts. The material was extracted by disrupting the cells with glass-beads, boiling in Laemmli buffer, separated by $4-12 \%$ Bis-Tris gels and stained with Coomassie blue.

Additional file 4: Minimal bactericial concentration of $E$. faecalis strains against antimicrobial peptides. Concentrations are expressed as $\mu \mathrm{g} / \mathrm{ml}$.
\end{abstract}

\section{Acknowledgements}

The authors thank Dr. Friedrich Feuerhake for help with electron microscopy, loana Toma and Dominique Wobser for excellent technical assistance. J.H. was supported by a grant of the German Ministry of Science and Education (ERA-Net PathoGenoMics 0313933).

\section{Author details}

${ }^{1}$ Center for Infectious Diseases and Travel Medicine, University Medical Center Freiburg, Germany. ${ }^{2}$ Division of Structural Biochemistry, Research Center Borstel, Leibniz-Center for Medicine and Biosciences, Germany.

\section{Authors' contributions}

$C T$ participated in the isolation and TLC analysis of glycolipids and LTA, the design and interpretation of the experiments, made the statistical analysis, and drafted the manuscript. IS performed the cell culture assays, autolysis assay and hydrophobicity assay. YB carried out the biofilm assay and participated in the molecular genetic studies. AK performed the opsonophagocytic killing assay and the mouse infection model. PSC performed the biochemical analysis of glycolipids and LTA. EG participated in the draft of the manuscript. OH participated in the biochemical analysis of the glycolipids and LTA and the draft of manuscript. JH participated in the design, coordination and interpretation of the study, and the draft of the manuscript. All authors read and approved the final manuscript.

Received: 22 October 2010 Accepted: 6 April 2011

Published: 6 April 2011

\section{References}

1. Weidenmaier C, Peschel A: Teichoic acids and related cell-wall glycopolymers in Gram-positive physiology and host interactions. Nat Rev Microbiol 2008, 6(4):276-287.

2. Theilacker C, Kaczynski Z, Kropec A, Sava I, Ye L, Bychowska A, Holst O, Huebner J: Serodiversity of Opsonic Antibodies against Enterococcus faecalis -Glycans of the Cell Wall Revisited. PLOS ONE 2011, 6(3):e17839. 
3. Teng F, Singh KV, Bourgogne A, Zeng J, Murray BE: Further characterization of the epa gene cluster and Epa polysaccharides of Enterococcus faecalis. Infect Immun 2009, 77(9):3759-3767.

4. Theilacker C, Kaczynski Z, Kropec A, Fabretti F, Sange T, Holst O, Huebner J: Opsonic antibodies to Enterococcus faecalis strain 12030 are directed against lipoteichoic acid. Infect Immun 2006, 74(10):5703-5712.

5. Theilacker C, Sanchez-Carballo P, Toma I, Fabretti F, Sava I, Kropec A Holst O, Huebner J: Glycolipids are involved in biofilm accumulation and prolonged bacteraemia in Enterococcus faecalis. Mol Microbiol 2009, 71(4):1055-1069

6. Wikström M, Xie J, Bogdanov M, Mileykovskaya E, Heacock P, Wieslander A, Dowhan W: Monoglucosyldiacylglycerol, a foreign lipid, can substitute for phosphatidylethanolamine in essential membrane-associated functions in Escherichia coli. J Biol Chem 2004, 279(11):10484-10493.

7. Edman M, Berg S, Storm P, Wikstrom M, Vikstrom S, Ohman A, Wieslander A: Structural features of glycosyltransferases synthesizing major bilayer and nonbilayer-prone membrane lipids in Acholeplasma laidlawii and Streptococcus pneumoniae. J Biol Chem 2003, 278(10):8420-8428.

8. Vikström S, Li L, Wieslander A: The nonbilayer/bilayer lipid balance in membranes. Regulatory enzyme in Acholeplasma laidlawii is stimulated by metabolic phosphates, activator phospholipids, and double-stranded DNA. J Biol Chem 2000, 275(13):9296-9302.

9. Campbell J, Davies G, Bulone V, Henrissat B: A classification of nucleotidediphospho-sugar glycosyltransferases based on amino acid sequence similarities. Biochem J 1998, 329(Pt 3):719.

10. Rahman O, Dover LG, Sutcliffe IC: Lipoteichoic acid biosynthesis: two steps forwards, one step sideways? Trends Microbiol 2009, 17(6):219-225.

11. Neuhaus FC, Baddiley J: A continuum of anionic charge: structures and functions of D-alanyl-teichoic acids in gram-positive bacteria. Microbiol Mol Biol Rev 2003, 67(4):686-723.

12. Fedtke I, Mader D, Kohler T, Moll H, Nicholson G, Biswas R, Henseler K, Götz F, Zähringer U, Peschel A: A Staphylococcus aureus ypfP mutant with strongly reduced lipoteichoic acid (LTA) content: LTA governs bacterial surface properties and autolysin activity. Mol Microbiol 2007, 65(4):1078-1091.

13. Grundling A, Schneewind $O$ : Genes required for glycolipid synthesis and lipoteichoic acid anchoring in Staphylococcus aureus. J Bacterio/ 2007, 189(6):2521-2530.

14. Berg S, Edman M, Li L, Wikstrom M, Wieslander A: Sequence properties of the 1,2-diacylglycerol 3-glucosyltransferase from Acholeplasma laidlawii membranes. Recognition of a large group of lipid glycosyltransferases in eubacteria and archaea. J Biol Chem 2001, 276(25):22056-22063.

15. Webb AJ, Karatsa-Dodgson M, Grundling A: Two-enzyme systems for glycolipid and polyglycerolphosphate lipoteichoic acid synthesis in Listeria monocytogenes. Mol Microbiol 2009, 74(2):299-314.

16. Kiriukhin MY, Debabov DV, Shinabarger DL, Neuhaus FC: Biosynthesis of the glycolipid anchor in lipoteichoic acid of Staphylococcus aureus RN4220: role of YpfP, the diglucosyldiacylglycerol synthase. J Bacteriol 2001, 183(11):3506-3514

17. Jorasch P, Wolter FP, Zähringer U, Heinz E: A UDP glucosyltransferase from Bacillus subtilis successively transfers up to four glucose residues to 1,2diacylglycerol: expression of ypfP in Escherichia coli and structural analysis of its reaction products. Mol Microbiol 1998, 29(2):419-430.

18. Doran KS, Engelson EJ, Khosravi A, Maisey HC, Fedtke I, Equils O, Michelsen KS, Arditi M, Peschel A, Nizet V: Blood-brain barrier invasion by group B Streptococcus depends upon proper cell-surface anchoring of lipoteichoic acid. J Clin Invest 2005, 115(9):2499-2507.

19. Fischer W: Bacterial phosphoglycolipids and lipoteichoic acids. In Handbook of Lipid Research. Volume 6. Edited by: Hanahan DJ. New York: Plenum Press; 1990:123-234.

20. Mohamed JA, Huang DB: Biofilm formation by enterococci. J Med Microbiol 2007, 56(Pt 12):1581-1588

21. Mohamed JA, Huang W, Nallapareddy SR, Teng F, Murray BE: Influence of origin of isolates, especially endocarditis isolates, and various genes on biofilm formation by Enterococcus faecalis. Infect Immun 2004, 72(6):3658-3663

22. Bos R, van der Mei HC, Busscher HJ: Physico-chemistry of initial microbial adhesive interactions-its mechanisms and methods for study. FEMS Microbiol Rev 1999, 23(2):179-230.
23. Courtney HS, Ofek I, Penfound T, Nizet V, Pence MA, Kreikemeyer B, Podbielski A, Podbielbski A, Hasty DL, Dale JB: Relationship between expression of the family of $\mathrm{M}$ proteins and lipoteichoic acid to hydrophobicity and biofilm formation in Streptococcus pyogenes. PLOS ONE 2009, 4(1):e4166.

24. Fabretti F, Theilacker C, Baldassarri L, Kaczynski Z, Kropec A, Holst O, Huebner J: Alanine esters of enterococcal lipoteichoic acid play a role in biofilm formation and resistance to antimicrobial peptides. Infect Immun 2006, 74(7):4164-4171.

25. Gross M, Cramton SE, Götz F, Peschel A: Key role of teichoic acid net charge in Staphylococcus aureus colonization of artificial surfaces. Infect Immun 2001, 69(5):3423-3426.

26. Neu TR: Significance of bacterial surface-active compounds in interaction of bacteria with interfaces. Microbiol Rev 1996, 60(1):151-166.

27. La Carbona S, Sauvageot N, Giard JC, Benachour A, Posteraro B, Auffray Y, Sanguinetti M, Hartke A: Comparative study of the physiological roles of three peroxidases (NADH peroxidase, Alkyl hydroperoxide reductase and Thiol peroxidase) in oxidative stress response, survival inside macrophages and virulence of Enterococcus faecalis. Mol Microbiol 2007, 66(5):1148-1163.

28. Sava IG, Zhang F, Toma I, Theilacker C, Li B, Baumert TF, Holst O, Linhardt RJ, Huebner J: Novel interactions of glycosaminoglycans and bacterial glycolipids mediate binding of enterococci to human cells. J Biol Chem 2009, 284(27):18194-18201.

29. Peschel A, Jack RW, Otto M, Collins LV, Staubitz P, Nicholson G, Kalbacher H, Nieuwenhuizen WF, Jung G, Tarkowski A, et al: Staphylococcus aureus resistance to human defensins and evasion of neutrophil killing via the novel virulence factor MprF is based on modification of membrane lipids with I-lysine. J Exp Med 2001, 193(9):1067-1076.

30. Qin X, Singh KV, Xu Y, Weinstock GM, Murray BE: Effect of disruption of a gene encoding an autolysin of Enterococcus faecalis OG1RF. Antimicrob Agents Chemother 1998, 42(11):2883-2888.

31. Reid G, Cuperus PL, Bruce AW, van der Mei HC, Tomeczek L, Khoury AH, Busscher HJ: Comparison of contact angles and adhesion to hexadecane of urogenital, dairy, and poultry lactobacilli: effect of serial culture passages. Appl Environ Microbiol 1992, 58(5):1549-1553.

32. Hufnagel M, Koch $S$, Creti $R$, Baldassarri L, Huebner J: A putative sugarbinding transcriptional regulator in a novel gene locus in Enterococcus faecalis contributes to production of biofilm and prolonged bacteremia in mice. J Infect Dis 2004, 189(3):420-430.

33. Huebner J, Wang Y, Krueger WA, Madoff LC, Martirosian G, Boisot S, Goldmann DA, Kasper DL, Tzianabos AO, Pier GB: Isolation and chemical characterization of a capsular polysaccharide antigen shared by clinical isolates of Enterococcus faecalis and vancomycin-resistant Enterococcus faecium. Infect Immun 1999, 67(3):1213-1219.

34. Callegan MC, Jett BD, Hancock LE, Gilmore MS: Role of hemolysin BL in the pathogenesis of extraintestinal Bacillus cereus infection assessed in an endophthalmitis model. Infect Immun 1999, 67(7):3357-3366.

35. Arnaud M, Chastanet A, Debarbouille M: New vector for efficient allelic replacement in naturally nontransformable, low-GC-content, grampositive bacteria. Appl Environ Microbiol 2004, 70(11):6887-6891.

doi:10.1186/1471-2180-11-67

Cite this article as: Theilacker et al:: Deletion of the glycosyltransferase bgs $B$ of Enterococcus faecalis leads to a complete loss of glycolipids from the cell membrane and to impaired biofilm formation. $B M C$ Microbiology 2011 11:67. 